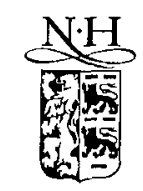

\title{
Reflectionless tunneling through a double-barrier NS junction
}

\author{
J.A. Melsen*, C.W.J. Beenakker \\ Instituut Lorentz Unversity of Leiden PO Box 95062300 RA Leiden The Netherlands
}

\begin{abstract}
The resistance is computed of an $\mathrm{NI}_{1} \mathrm{NI}_{2} \mathrm{~S}$ junction, where $\mathrm{N}$ is the normal metal, $\mathrm{S}$ the superconductor, and $\mathrm{I}_{2}$ the insulator or tunnel barrier (transmission probability per mode $\Gamma_{1}$ ) The ballistic case is considered, as well as the case that the region between the two barriers contains disorder (mean free path $l$, barrier separation $L$ ) It is found that the resistance at fixed $\Gamma_{2}$ shows a minimum as a function of $\Gamma_{1}$, when $\Gamma_{1} \approx \sqrt{2} \Gamma_{2}$, provided $l \gtrsim \Gamma_{2} L$ The minimum is explained in terms of the appearance of transmission eggenvalues close to one, analogous to the "reflectionless tunneling" through a NIS junction with a disordered normal region The theory is supported by numerical simulations
\end{abstract}

\section{Introduction}

Reflectionless tunneling is a novel quantum interference effect which occurs when dissipative normal current is converted into dissipationless supercurrent at the interface between a normal metal $(\mathrm{N})$ and a superconductor (S) [1] Experimentally, the effect is observed as a peak in the differentral conductance around zero voltage or around zero magnetic field [2] Its name refers to the fact that, for full-phase coherence, the Andreev-reflected quasiparticle can tunnel through the potential barricr at the NS interface without suffering reflections (The potential barrier can be the insulator (I) in an NIS junction, or the Schottky barrier in a semiconductor-superconductor junction ) Application of a voltage or magnetic field destroys the phase coherence between electrons and holes, and thus reduces the conductance of the junction We now have a good theoretical understanding of the effect, based on a combination of numerical $[3,4]$, and analytical work [5-10] The basic requirement for reflectionless tunneling is that the normal region has a resistance which is larger than the resistance of the interface In that case the disorder is able to open a fraction of the tunneling channels, $1 \mathrm{e}$ it induces the appearance of transmission eigenvalues close to one [10] As a result of these open channels, the resistance has a linear dependence on the transparency of the interface, instead of the quadratic de-

* Corresponding author pendence expected for Andreev reflection [11] (which is a two-particle process)

The purpose of this work is to present a study of reflectionless tunneling in its simplest form, when the resistance of the normal metal is due to a second tunnel barrier, in series with the barrier at the NS interface This allows an exact calculation, which shows many of the features of the more complicated case when the resistance of the normal region is due to disorder Furthermore, the double-barrier geometry provides an experimentally realizable model system, for example, in tunnelıng from an STM into a superconductor via a metal particle [12]

The outline of this paper is as follows In Section 2 we consider the problem of a $\mathrm{NI}_{1} \mathrm{NI}_{2} \mathrm{~S}$ junction without disorder We compute the resistance of the junction as a function of the transmission probabilities per mode $\Gamma_{1}$ and $\Gamma_{2}$ of the two barriers The resistance at fixed $\Gamma_{2}$ shows a minumum as a function of $\Gamma_{1}$ when $\Gamma_{1} \approx \sqrt{2} \Gamma_{2} \equiv \Gamma$ The resistance in the minimum depends linearly on $1 / \Gamma$, in contrast to the quadratic dependence in the case of a single barrier In Section 3 we apply a recent scaling theory [9], to find the influence on the resistance minimum of disorder in the region between the barriers (length $L$, mean free path $l$ ) The resistance minımum persists as long as $l \gtrsim \Gamma L$ In the diffusive regime $(l \ll L)$, our results agiee with a previous Green's function calculation by Volkov et al [7] The analytical results are supported by numerical simulations, usingthe recursive Green's function technique [13] We conclude in Section 4 


\section{NINIS junction without disorder}

We consider a $\mathrm{NI}_{1} \mathrm{NI}_{2} \mathrm{~S}$ junction, where $\mathrm{N}$ is the normal metal, $\mathrm{S}$ is the superconductor, and $\mathrm{I}_{t}$ the insulator or tunnel barrier (see inset of Fig. 1). The transmission probability per mode of $\mathrm{I}_{l}$ is denoted by $\Gamma_{l}$. For simplicity, we neglect the mode dependence of $\Gamma_{l}$. In this section, we assume ballistic motion between the barriers. (The effect of disorder in the normal region is considered in Section 3.) A straightforward calculation yields the transmission probabilities $T_{n}$ of the two barriers in series,

$T_{n}=\left(a+b \cos \phi_{n}\right)^{-1}$,

where

$a=1+\frac{2-\Gamma_{1}-\Gamma_{2}}{\Gamma_{1} \Gamma_{2}}$,

$b=\frac{2\left(1-\Gamma_{1}\right)^{1 / 2}\left(1-\Gamma_{2}\right)^{1 / 2}}{\Gamma_{1} \Gamma_{2}}$,

and $\phi_{n}$ is the phase accumulated between the barriers by mode $n=1,2, \ldots, N$ (with $N$ the number of propagating modes at the Fermi level). If we substitute $\Gamma_{l}=1 / \cosh ^{2} \alpha_{l}$ $\left(\alpha_{2} \geqslant 0\right)$, the coefficients $a$ and $b$ can be rewritien as

$a=\frac{1}{2}+\frac{1}{2} \cosh 2 \alpha_{1} \cosh 2 \alpha_{2}$,

$b=\frac{1}{2} \sinh 2 \alpha_{1} \sinh 2 \alpha_{2}$.

Since the transmission matrix $t$ is diagonal, the transmission probabilities $T_{n}$ are identical to the eigenvalues of $t t^{\dagger}$.

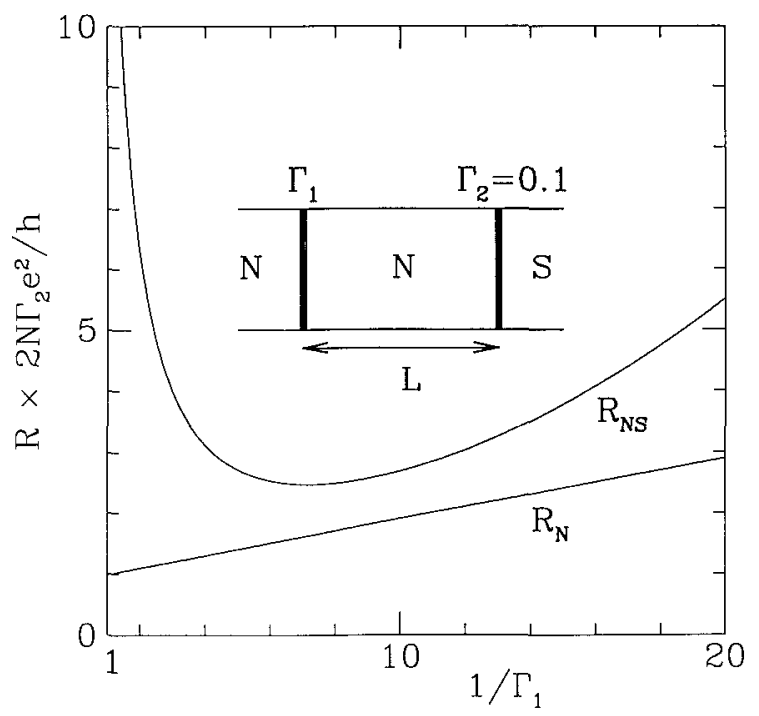

Fig. 1. Dependence of the resistances $R_{\mathrm{N}}$ and $R_{\mathrm{NS}}$ of ballistic NININ and NINIS structures, respectively, on barrier transparency $\Gamma_{1}$, while transparancy $\Gamma_{2}=0.1$ is kept fixed [computed from Eqs.(2.6) and (2.7)]. The inset shows the NINIS structure considered.
We use the general relationship between the conductance $G_{\mathrm{NS}} \equiv G_{\mathrm{NINIS}}$ of the NINIS junction and the transmission eigenvalues of the normal region [14],

$G_{\mathrm{NS}}=\frac{4 e^{2}}{h} \sum_{n=1}^{N} \frac{T_{n}^{2}}{\left(2-T_{n}\right)^{2}}$,

which is the analogue of the Landauer formula,

$G_{\mathrm{N}}=\frac{2 e^{2}}{h} \sum_{n=1}^{N} T_{n}$

for the conductance $G_{N} \equiv G_{\text {NININ }}$ in the normal state. We assume that $L \gg \lambda_{\mathrm{F}}\left(\lambda_{\mathrm{F}}\right.$ is the Fermi wavelength) and $N \Gamma_{1} \gg 1$, so that the conductance is not dominated by a single resonance. In this case, the phases $\phi_{n}$ are distributed uniformly in the interval $(0,2 \pi)$ and we may replace the summations in Eqs. (2.4) and (2.5) by integrals over $\phi: \sum_{n=1}^{N} f\left(\phi_{n}\right) \rightarrow$ $(N / 2 \pi) \int_{0}^{2 \pi} \mathrm{d} \phi f(\phi)$. The result is

$$
\begin{aligned}
G_{\mathrm{NS}} & =\frac{4 e^{2} N}{h} \frac{\cosh 2 \alpha_{1} \cosh 2 \alpha_{2}}{\left(\cosh ^{2} 2 \alpha_{1}+\cosh ^{2} 2 \alpha_{2}-1\right)^{3 / 2}}, \\
G_{\mathrm{N}} & =\frac{4 e^{2} N}{h}\left(\cosh 2 \alpha_{1}+\cosh 2 \alpha_{2}\right)^{-1} .
\end{aligned}
$$

These expressions are symmetric in the indices 1 and 2; it does not matter which of the two barriers is closest to the superconductor.

In the same way we can compute the entire distribution of the transmission eigenvalues, $\rho(T) \equiv \sum_{n} \delta\left(T-T_{n}\right) \rightarrow$ $(N / 2 \pi) \int_{0}^{2 \pi} \mathrm{d} \phi \delta(T-T(\phi))$. Substituting $T(\phi)=(a+$ $b \cos \phi)^{-1}$ from Eq. (2.1), we find

$\rho(T)=\frac{N}{\pi T}\left(b^{2} T^{2}-(a T-1)^{2}\right)^{-1 / 2}$

for $(a+b)^{-1}<T<(a-b)^{-1}$.

In Fig. 1 we plot the resistance $R_{\mathrm{N}}=1 / G_{\mathrm{N}}$ and $R_{\mathrm{NS}}=$ $1 / G_{\mathrm{NS}}$, following from Eqs. (2.6) and (2.7). Notice that $R_{\mathrm{N}}$ follows Ohm's law,

$R_{\mathrm{N}}=\frac{h}{2 N e^{2}}\left(1 / \Gamma_{1}+1 / \Gamma_{2}-1\right)$,

as expected from classical considerations. In contrast, the resistance $R_{\mathrm{NS}}$ has a minimum if one of the $\Gamma$ 's is varied while keeping the other fixed. This resistance minimum cannot be explained by classical series addition of barrier resistances. If $\Gamma_{2} \ll 1$ is fixed and $\Gamma_{1}$ is varied, as in Fig. 1, the minimum occurs when $\Gamma_{1}=\sqrt{2} \Gamma_{2}$. The minimal resistance $R_{\mathrm{NS}}^{\mathrm{min}}$ is of the same order of magnitude as the resistance $R_{\mathrm{N}}$ in the normal state at the same value of $\Gamma_{1}$ and $\Gamma_{2}$. (For $\Gamma_{2} \ll 1, R_{\mathrm{NS}}^{\min }=1.52 R_{\mathrm{N}}$ ) In particular, we find that $R_{\mathrm{NS}}^{\min }$ depends linearly on $1 / \Gamma_{t}$, whereas for a single barrier $R_{\mathrm{NS}} \propto 1 / \Gamma^{2}$. 


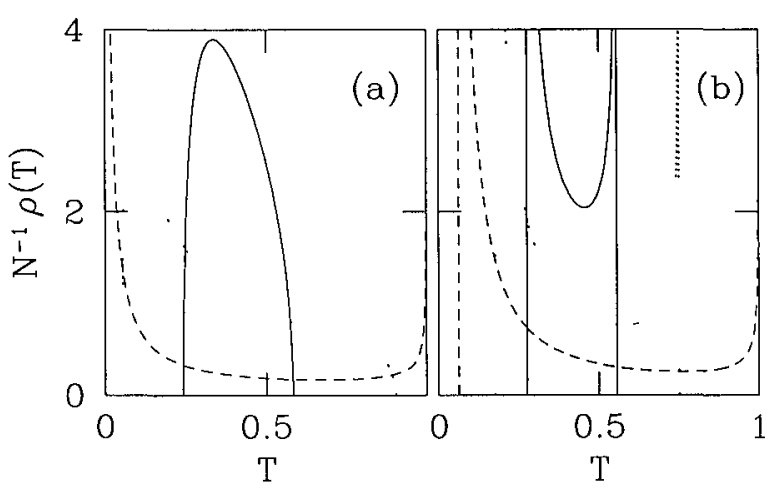

Fig. 2. Density of normal-state transmission eigenvalues for an NS junction with a potential barrier at the interface (transmission probability $\Gamma=0.4$ ) The left panel (a) shows the disorder-induced opening of tunneling channels in a NIS junction (solid curve: $s=0.04$, dotted: $s=0.4$, dashed $s=5$; where $s \equiv L / l$ ). The right panel (b) shows the opening of channels by a second tunnel barrier (transparancy $\Gamma^{\prime}$ ) in an NINIS junction (solid curve: $\Gamma^{\prime}=0.95$; dotted: $\Gamma^{\prime}=0.8$, dashed: $\Gamma^{\prime}=0.4$ ). The curves in (a) are computed from Ref. [9], the curves in (b) from Eq.(2.8). Notice the similarity of the dashed curves.

The linear dependence on the barrier transparency shows the qualitatıve similarity of a ballistic NINIS junction to a disordered NIS junction. To illustrate the similarity, we compare in Fig. 2 the densities of transmission eigenvalues through the normal region. The left panel is for an NIS junction (computed using the results of Ref. [9]), the right panel is for an NINIS junction (computed from Eq. (2.8)). In the NIS junction, disorder leads to a bimodal distribution $\rho(T)$, with a peak near zero transmission and another peak near unit transmission (dashed curve). A similar bimodal distribution appears in the ballistic NINIS junction for approximately equal transmission probabilities of the two barriers. There are also differences between the two cases: The NIS junction has a unimodal $\rho(T)$ if $L / l<1 / \Gamma$, while the NINIS junction has a bimodal $\rho(T)$ for any ratio of $\Gamma_{1}$ and $\Gamma_{2}$. In both cases, the opening of tunneling channels, i.e., the appearance of a peak in $\rho(T)$ near $T=1$, is the origin for the $1 / \Gamma$ dependence of the resistance.

\section{Effects of disorder}

Let us now investigate what happens to the resistance minimum if the region of length $L$ between the tunnel barriers contains impurities, with elastic mean free path $l$. We denote $s \equiv L / l$. When introducing disorder, it is necessary to consider ensemble-averaged quantities. To calculate the ensemble-averaged conductance $\left\langle G_{\mathrm{NS}}\right\rangle$, we need to know the density $\rho$ of the transmission eigenvalues $T_{n}$ as a function of $s$. It is convenient to work with the parameterization

$T_{n}=1 / \cosh ^{2} x_{t}, \quad x_{n} \geqslant 0$.
The density of the $x_{n}$ 's is defined by $\rho(x, s) \equiv\left\langle\sum_{n} \delta(x-\right.$ $\left.\left.x_{n}\right)\right\rangle$. From Eq. (2.1) we know that, for $s=0$ (no disorder),

$$
\begin{aligned}
\rho(x, 0) & =N \int_{0}^{2 \pi} \frac{\mathrm{d} \phi}{2 \pi} \delta(x-\operatorname{arccosh} \sqrt{a+b \cos \phi}) \\
& =\frac{N}{\pi} \sinh 2 x\left(b^{2}-\left(a-\cosh ^{2} x\right)^{2}\right)^{-1 / 2},
\end{aligned}
$$

for $\operatorname{arccosh} \sqrt{a-b} \equiv x_{\min }<x<x_{\max } \equiv \operatorname{arccosh} \sqrt{a+b}$.

For $s>0$ we obtain the density $\rho(x, s)$ from the integrodifferential equation [15]

$$
\begin{aligned}
& \frac{\partial}{\partial s} \rho(x, s)=-\frac{1}{2 N} \frac{\partial}{\partial x} \rho(x, s) \frac{\partial}{\partial x} \\
& \int_{0}^{\infty} \mathrm{d} x^{\prime} \rho\left(x^{\prime}, s\right) \ln \left|\sinh ^{2} x-\sinh ^{2} x^{\prime}\right|,
\end{aligned}
$$

which is the large $N$-limit of the scaling equation due to Dorokhov [16] and Mello et al. [17]. This equation describes the evolution of $\rho(x, s)$ when an infinitesimal slice of disordered material is added. With initial condition (3.2) it therefore describes a geometry where all disorder is on one side of the two tunnel barriers, rather than in between. In fact, only the total length $L$ of the disordered region matters, and not the location relative to the barriers. The argument is similar to that in Ref. [18]. The total transfer matrix $M$ of the normal region is a product of the transfer matrices of its constituents (barriers and disordered segments): $M=$ $M_{1} M_{2} M_{3} \ldots$. The probability distribution of $M$ is given by the convolution $p(M)=p_{1} * p_{2} * p_{3} * \cdots$ of the distributions $p_{\imath}$ of transfer matrices $M_{\imath}$. The convolution is defined as

$p_{I} * p_{J}(M)=\int \mathrm{d} M^{\prime} p_{I}\left(M M^{\prime-1}\right) p_{J}\left(M^{\prime}\right)$.

If for all parts $i$ of the system, $p_{t}\left(M_{i}\right)$ is a function of the eigenvalues of $M_{t} M_{l}^{\dagger}$ only, the convolution of the $p_{t}$ commutes [18]. The distributions $p$, are then called isotropic. A disordered segment (length $L$, width $W$ ) has an isotropic distribution if $L \gg W$. A planar tunnel barrier does not mix the modes, so a priori it does not have an isotropic distribution. However, if the mode dependence of the transmission probabilities is neglected (as we do here), it does not make a difference if we replace its distribution by an isotropic one. The commutativity of the convolution of isotropic distributions implies that the location of the tunnel barriers with respect to the disordered region does not affect $\rho(x, s)$. The systems in Figs. 3(a)-(c) then have identical statistical properties.

Once $\rho(x, s)$ is known, the conductances $\left\langle G_{\mathrm{NS}}\right\rangle$ and $\left\langle G_{\mathrm{N}}\right\rangle$ can be determined from

$$
\begin{aligned}
& \left\langle G_{\mathrm{NS}}\right\rangle=\frac{4 e^{2}}{h} \int_{0}^{\infty} \mathrm{d} x \frac{\rho(x, s)}{\cosh ^{2} 2 x}, \\
& \left\langle G_{\mathrm{N}}\right\rangle=\frac{2 e^{2}}{h} \int_{0}^{\infty} \mathrm{d} x \frac{\rho(x, s)}{\cosh ^{2} x},
\end{aligned}
$$


a
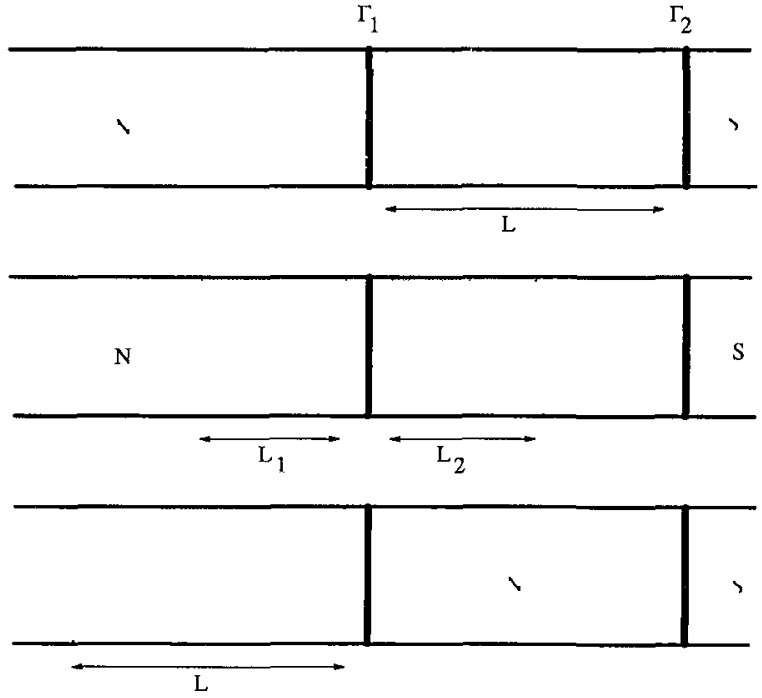

Fig 3 The systems $a, b$, and $c$ are statistically equivalent, if the transfer matrices of each of the two barriers (solid vertical lines) and the disordered regions (shaded areas, $L_{1}+L_{2}=L$ in case b) have isotropic distributions, in that case, the position of the disorder with respect to the barriers does not affect the eigenvalue density $\rho(x, s)$

where we have substituted Eq (3 1) into Eqs (2 4) and (2 5) In Ref [9] a general solution to the evolution equation was obtained for arbitrary initial condition It was shown that $\mathrm{Eq}$ (3 3) can be mapped onto Euler's equation of hydrodynamics

$\frac{\partial}{\partial s} U(\zeta, s)+U(\zeta, s) \frac{\partial}{\partial \zeta} U(\zeta, s)=0$

by means of the substitution

$U(\zeta, s)=\frac{\sinh 2 \zeta}{2 N} \int_{0}^{\infty} \mathrm{d} x^{\prime} \frac{\rho\left(x^{\prime}, s\right)}{\sinh ^{2} \zeta-\sinh ^{2} x^{\prime}}$

Here, $U \equiv U_{x}+{ }_{1} U_{y}$ and $\zeta \equiv x+1 y$ Eq (3 7) describes the velocity field $U(\zeta, s)$ of a $2 \mathrm{D}$ ideal fluid at constant pressure in the $x-y$ plane Its solution is ${ }^{1}$

$U(\zeta, s)=U_{0}(\zeta-s U(\zeta, s))$

in terms of the initial value $U_{0}(\zeta) \equiv U(\zeta, 0)$ The probability distribution $\rho(x, s)$ follows from the velocity field by inversion of $\mathrm{Eq}(38)$,

$\rho(x, s)=\frac{2 N}{\pi} U_{y}(x-1 \varepsilon, s)$

where $\varepsilon$ is a positive infinitesimal

${ }^{1}$ The implicit equation (39) has multiple solutions in the entire complex plane, we need the solution for which both $\zeta$ and $\zeta-$ $s U(\zeta, s)$ lie in the strip between the lines $y=0$ and $y=-\pi / 2$

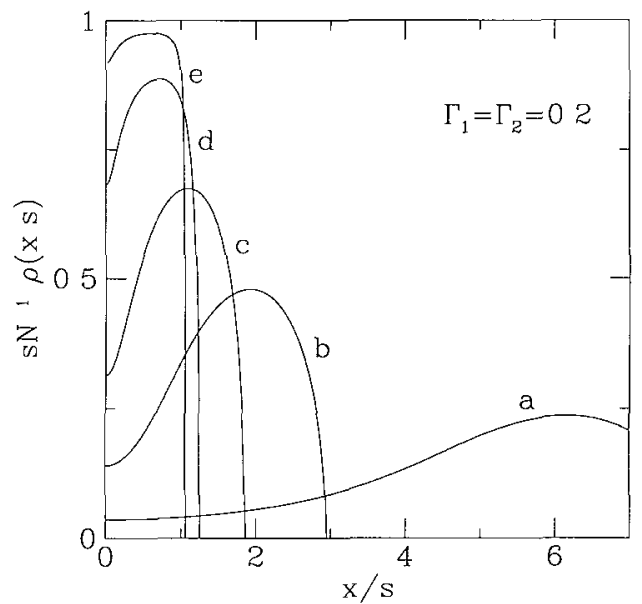

Fig 4 Eigenvalue density $\rho(x, s)$ as a function of $x$ (in units of $s \equiv L / l$ ) for $\Gamma_{1}=\Gamma_{2}=02$ Curves a,b,c,d, and e are for $s=05,2,5,20,100$, respectively In the special case of equal tunnel barriers, open channels exist already in the absence of disorder

In our case, the initial velocity field [from Eqs $(32)$ and (3 8)] is

$U_{0}(\zeta)=-\frac{1}{2} \sinh 2 \zeta\left[\left(\cosh ^{2} \zeta-a\right)^{2}-b^{2}\right]^{-1 / 2}$

The resulting density ( 310 ) is plotted in Fig 4 for $\Gamma_{1}=$ $\Gamma_{2} \equiv \Gamma$ and several disorder strengths The region near $x=$ 0 is of importance for the conductance (since $x$ near zero corresponds to near-unit transmission) The number $N_{\text {open }} \equiv$ $\rho(0, s)$ is an estimate for the number of transmission eigenvalues close to 1 (the so-called "open channels" [19]) In the absence of disorder, $N_{\text {opcn }}$ is non-zero only if $\Gamma_{1} \approx$ $\Gamma_{2}$ (then $a-b=1 \Rightarrow x_{\min }=0$ ) From Eq (32) we find $N_{\text {open }}=N \Gamma / \pi$ for $s=0$ and $\Gamma_{1}=\Gamma_{2} \equiv \Gamma \ll 1$ Adding disorder reduces the number of open channels If $\Gamma_{1} \neq \Gamma_{2}$ there are no open channels for $s=0\left(x_{\mathrm{mm}}>0\right)$ Disorder then has the effect of increasing $N_{\text {open }}$, such that $N_{\text {open }} \approx N / s$ if $\left(\Gamma_{1}+\Gamma_{2}\right) s \gg 1$ The disorder-1nduced opening of channels was studied in Refs $[9,10]$ for the case of a single-tunnelbarrer

To test our analytical results for the eigenvalue density $\rho(x, s)$, we have carried out numerical simulations, similar to those reported in Ref [9] The sample was modeled by a tight-binding Hamiltonian on a square lattıce with lattice constant $a$ The tunnel barriers were accounted for by assigning a non-random potential energy $U_{\mathrm{B}}=23 E_{\mathrm{F}}$ to a single row of sites at both ends of the lattice, which corresponds to a mode-averaged barrier transparency $\Gamma_{1}=\Gamma_{2}=018$ The Fermi energy was chosen at $15 u_{0}$, with $u_{0}=\hbar^{2} / 2 m a^{2}$ Disorder was introduced by randomly assigning a value between $\pm \frac{1}{2} U_{\mathrm{D}}$ to the on-site potential of the lattice points between the barriers The disorder strength $U_{\mathrm{D}}$ was varied between 0 and $15 u_{0}$, corresponding to $s$ between 0 and $117 \mathrm{We}$ considered geometries with both a square disordered region 


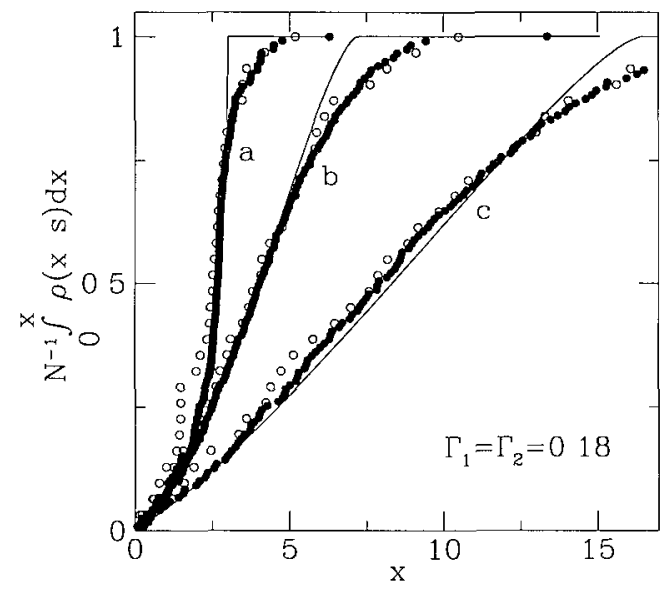

Fig 5 Comparison between theory and simulation of the integrated eigenvalue density for $\Gamma_{1}=\Gamma_{2}=018$ The labels a, b, c indicate, respectively, $s=0,3,117$ Solıd curves are from Eq (39), data points are the $x_{n}$ 's from the simulation plotted in ascending order versus $n / N$ Filled data points are for a square geometry, open points are for an aspect ratio $L / W=38$

$(285 \times 285$ sites, $N=119)$ and a rectangular one $(285 \times 75$ sites, $N=31$ ), to test the geometry dependence of our results In Fig 5, we compare the integrated ergenvalue density $v(x, s) \equiv N^{-1} \int_{0}^{x} \mathrm{~d} x^{\prime} \rho\left(x^{\prime}, s\right)$ with the numerical results

The quantity $v(x, s)$ follows directly from our simulations by plotting the $x_{n}$ 's in ascending order versus $n / N \equiv v$ We want to sample $v(x, s)$ at many points along the $x$-axis, so we need $N$ large Since the $x_{n}$ 's are self-averaging (fluctuations are of the order of $1 / N$ ), it is not necessary to average over many samples The data shown in Fig 5 are from a single realization of the impurity potential There is good agreement with the analytical results No geometry dependence is observed, which indicates that the restriction $L \gg W$ of $\mathrm{Eq}$ (3 3) can be relaxed to a considerable extent

Using Eqs (35) and (38), the average conductance $\left\langle G_{\mathrm{NS}}\right\rangle$ can be directly expressed in terms of the velocity field,

$\left\langle G_{\mathrm{NS}}\right\rangle=\frac{2 N e^{2}}{h} \lim _{\zeta \rightarrow-1 \pi / 4} \frac{\partial}{\partial \zeta} U(\zeta, s)$

for $\zeta \rightarrow-1 \pi / 4, U \rightarrow 1 U_{y}, U_{y}>0$ The implicit solution (39) then takes the form

$\phi \sqrt{(2 a+\sin \phi-1)^{2}-4 b^{2}}=2 s \cos \phi$,

where $\phi \equiv 2 s U_{y} \in[0, \pi / 2]$ We now use that

$\left.\frac{\partial}{\partial \zeta} U(\zeta, s)\right|_{\zeta=-1 \pi / 4}=-\left[\frac{\partial}{\partial s} U\left(-\frac{1 \pi}{4}, s\right)\right] / U\left(-\frac{1 \pi}{4}, s\right)$

[see Eq (3 7)] Combinıng Eqs (3 12) and (3 13) we find

$\left\langle G_{\mathrm{NS}}\right\rangle=\frac{2 N e^{2}}{h}(s+1 / Q)$

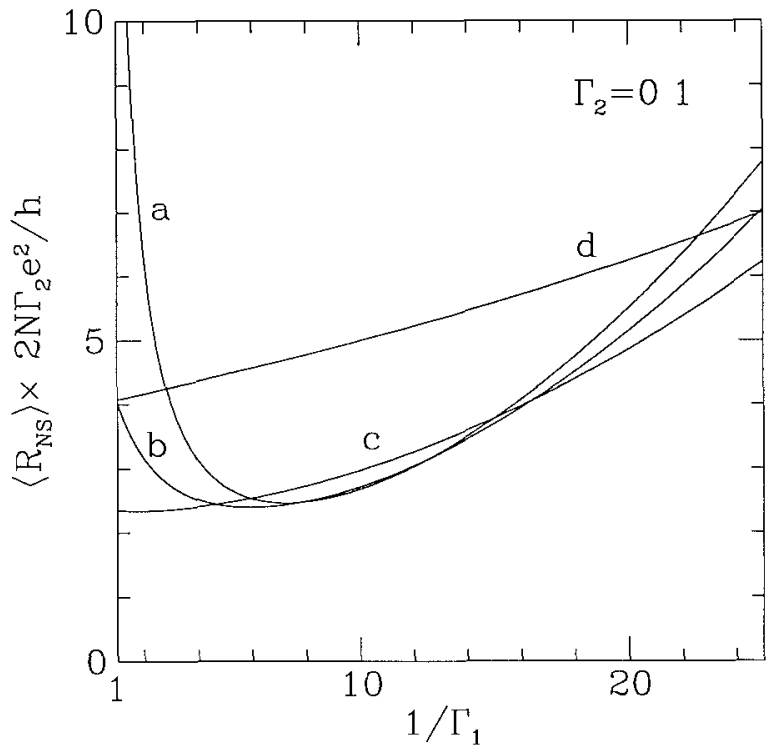

Fig 6 Dependence of the ensemble averaged resistance $\left\langle R_{\mathrm{NS}}\right\rangle$ for a disordered NINIS junction on barrer transparancy $\Gamma_{1}$, while $\Gamma_{2}=01$ is kept fixed [computed from Eqs (3 14) and (3 15)] Curves a, b, c, d are for $s=027,30$, respectively The resistance minımum persists for small disorder

where the effective tunnel rate $Q$ is given in terms of the angle $\phi$ in Eq (3 13) by

$$
\begin{aligned}
& Q=\frac{\phi}{s \cos \phi} \\
& \times\left(\sin \phi+\frac{\phi^{2}}{4 s^{2}}\left[\sin \phi+\left(1-2 / \Gamma_{1}\right)\left(1-2 / \Gamma_{2}\right)\right]\right)
\end{aligned}
$$

Eqs (3 13)-(3 15) completely determıne the conductance of a double-barrier NS-junction containing disorder

In Fig 6 , we plot $\left\langle R_{\mathrm{NS}}\right\rangle$ for several values of the disorder, keeping $\Gamma_{2}=01$ fixed and varying the transparency of barrier 1 For weak disorder $\left(\Gamma_{2} s \ll 1\right)$, the resistance mın1 mum is retained, but its location moves to larger values of $\Gamma_{1}$ On increasing the disorder, the minimum becomes shallower and eventually disappears In the regime of strong disorder $\left(\Gamma_{2} s \geqslant 1\right)$, the resistance behaves nearly Ohmic

We stress that these results hold for arbitrary $s \equiv L / l$, all the way from the ballistic into the diffusive regime Volkov et al [7] have computed $\left\langle G_{\mathrm{NS}}\right\rangle$ in the diffusive limit $s \gg 1$ In that limit our Eqs (3 13) and (3 15) take the form

$$
\frac{s \cos \phi}{\phi}=\frac{1}{\Gamma_{1}} \sqrt{1-\left(\frac{\phi}{\Gamma_{2} s}\right)^{2}}+\frac{1}{\Gamma_{2}} \sqrt{1-\left(\frac{\phi}{\Gamma_{1} s}\right)^{2}}
$$

$\frac{1}{Q}=\sum_{t=1}^{2} \frac{1}{\Gamma_{l}}\left[1-\left(\frac{\phi}{\Gamma_{l} s}\right)^{2}\right]^{-1 / 2}$, 
in precise agreement with Ref [7] Nazarov's circuit theory [10], which is equivalent to the Green's function theory of Ref [7], also leads to this result for $\left\langle G_{\mathrm{NS}}\right\rangle$ in the diffusive regime

Two limitıng cases of Eqs ( 316$)$ and (3 17) are of particular interest For strong barriers, $\Gamma_{1}, \Gamma_{2} \ll 1$, and stiong disorder, $s \gg 1$, one has the two asymptotic formulas

$$
\begin{aligned}
& \left\langle G_{\mathrm{NS}}\right\rangle=\frac{2 N e^{2}}{h} \frac{\Gamma_{1}^{2} \Gamma_{2}^{2}}{\left(\Gamma_{1}^{2}+\Gamma_{2}^{2}\right)^{3 / 2}}, \quad \text { if } \Gamma_{1}, \Gamma_{2} \ll 1 / s, \\
& \left\langle G_{\mathrm{NS}}\right\rangle=\frac{2 N e^{2}}{h}\left(s+1 / \Gamma_{1}+1 / \Gamma_{2}\right)^{-1}, \quad \text { if } \Gamma_{1}, \Gamma_{2} \gg 1 / s
\end{aligned}
$$

Eq (3 18) coincides with Eq (26) in the limit $\alpha_{1}, \alpha_{2} \gg 1$ (recall that $\Gamma_{t} \equiv 1 / \cosh ^{2} \alpha_{t}$ ) This shows that the effect of disorder on the resistance minimum can be neglected as long as the resistance of the junction is dominated by the barriers In this case $\left\langle G_{\mathrm{NS}}\right\rangle$ depends linearly on $\Gamma_{1}$ and $\Gamma_{2}$ only if $\Gamma_{1} \approx \Gamma_{2}$ Eq (3 19) shows that if the disorder dominates, $\left\langle G_{\mathrm{NS}}\right\rangle$ has a linear $\Gamma$ dependence regardless of the relatıve magnitude of $\Gamma_{1}$ and $\Gamma_{2}$

\section{Conclusions}

In summary, we have derived an expression for the conductance of a ballistic NINIS junction in the limit $N \Gamma \gg 1$ that the tunnel resistance is much smaller than $h / e^{2}$ In this regime the double-barrier junction contains a large number of overlapping resonances, so that in the normal state the resistance depends monotonically on $1 / \Gamma$ In contrast, the NINIS junction shows a resistance minımum when one of the barrier transparencies is varied while the other is kept fixed The minimal resistance (at $\Gamma_{1} \simeq \Gamma_{2} \equiv \Gamma$ ) is proportional to $1 / \Gamma$, instead of the $1 / \Gamma^{2}$ dependence expected for two-particle tunneling into a superconductor This is similar to the reflectionless tunneling which occurs in an NIS junction Using the results of the ballistic junction, we have described the transition to a disordered NINIS junction by means of an evolution equation for the transmission eigenvalue density [9] We found that the resistance minimum is unaffected by disorder, as long as $l \gg L / \Gamma, 1 \mathrm{e}$, as long as the barrier resistance dominates the junction resistance As the disorder becomes more dominant, a transition to a monotonic $\Gamma$ dependence takes place In the limit of diffusive motion between the barriers, our results agree with Ref [7]

Throughout this paper we have assumed zero temperature, zero magnetic field, and infinitesimal applied voltage Each of these quantities is capable of destroying the phasc coherence between the electrons and the Andreev-reflected holes, which is responsible for the resistance minımum As far as the temperature $T$ and voltage $V$ are concerned, we require $k_{\mathrm{B}} T, e V \ll \hbar / \tau_{\mathrm{dwcll}}$ for the appearance of a resistance minimum, where $\tau_{\text {dwell }}$ is the dwell time of an elcctron in the region between the two barriers For a ballistic NINIS junction, we have $\tau_{\text {dwell }} \sim L / v_{\mathrm{F}} \Gamma$, while for a disordered junction $\tau_{\mathrm{dwell}} \sim L^{2} / v_{\mathrm{F}} \Gamma l$ s larger by a factor $L / l$ It follows that the condition on temperature and voltage becomes more restrictive if the disorder increases, even if the resistance remains dominated by the barriers As far as the magnetic field $B$ is concerned, we require $B \ll h / e S$ (with $S$ the area of the juncton perpendicular to $B$ ) if the motion between the barriers is diffusive For ballistic motion the trajectories enclose no flux, so no magetic field dependence is expected

A possible experiment to verify our results might be scanning tunneling microscopy of a metal particle on top of a superconductung substrate [12] The metal-superconductor interface has a fixed tunnel probability $\Gamma_{2}$ The probability $\Gamma_{1}$ for an electron to tunnel from STM to particle can be controlled by varying the distance (Volkov has recently analyzed this geometry in the regime that the motion from STM to particle is diffusive rather than by tunneling [20] ) Another possibility is to create an NINIS junction using a two-dimensional electron gas in contact with a superconductor The tunnel barriers could then be implemented by means of two gate electrodes In this way both transparancles might be tuned independently

\section{Acknowledgement}

This research was motivated by a discussion with $\mathrm{D}$ Esteve, which is gratefully acknowledged Financial support was provided by the "Nederlandse organtsatie voor Wetenschappelyk Onderzoek" (NWO) by the "Strchting voor Fundamenteel Onderzoek der Materie" (FOM), and by the "Human Capital and Mobility" programme of the European Communty

\section{References}

[1] For a review of quantum interference effects in NS junctions, see C W J Beenakker, in Mesoscopic Quantum Physics, eds E Akkermans, G Montambaux and J-L Pichard (NorthHolland, Amsterdam) to be published

[2] A Kastalsky, A W Kleınsasser, L H Greene, R Bhat, F P Milliken and J P Ilarbison, Phys Rev Lett 67 (1991) 3026

[3] I K Marmorkos, C W J Beenakker and R A Jalabert, Phys Rev B 48 (1993) 2811

[4] Y Takane and H Ebisawa, J Phys Soc Japan 62 (1993) 1844

[5] B J van Wees, P de Vries, P Magnee and T M Klapwijk, Phys Rev Lett 69 (1992) 510

[6] Y Takane and H Ebisawa, J Phys Soc Japan 61 (1992) 3466

[7] A F Volkov, A V Zaitsev and T M Klapwıjk, Physica C 210 (1993) 21

[8] F W J Hekkıng and Yu V Nazarov, Phys Rev Lett 71 (1993) 1625, Phys Rev B 49 (1994) 6847 
[9] C W J Beenakker, B Rejaeı and J A Melsen, Phys Rev Lett 72 (1994) 2470

[10] Yu V Nazarov, Phys Rev Lett 73 (1994) 134

[11] A L Shelankov, P1s'ma Zh Eksp Teor F1z 32 (1980) 122 [JETP Lett 32 (1980) 111]

[12] D R Heslinga, SE Shafranjuk, $H$ van Kempen and T M Klapw1jk, Phys Rev B 49 (1994) 10484

[13] H U Baranger, D P DiVincenzo, R A Jalabert and A D Stone, Phys Rev B 44 (1991) 10637 The computer code for the recursive Green's function calculation was kindly made avalable to us by Dr Jalabert
[14] C W J Beenakker, Phys Rev B 46 (1992) 12841

[15] P A Mello and J-L Pichard, Phys Rev B 40 (1989) 5276

[16] ON Dorokhov, Pis'ma Zh Eksp Teor Fiz 36 (1982) 259 [JETP Lett 36 (1982) 318]

[17] P A Mello, P Pereyra and N Kumar, Ann Phys (NY) 181 (1988) 290

[18] C W J Beenakker and J A Melsen, Phys Rev B 50 (1994) 2450

[19] Y Imry, Europhys Lett 1 (1986) 249

[20] A F Volkov, Phys Lett A 187 (1994) 404 